\title{
ON AN INTERPOLATION INEQUALITY AND ITS APPLICATIONS TO NONLINEAR ELLIPTIC EQUATIONS
}

\author{
NEIL S. TRUDINGER
}

\begin{abstract}
We discuss the application of a nonlinear interpolation inequality involving Hölder norms to the estimation of derivatives of solutions of second order nonlinear elliptic equations.
\end{abstract}

In this note we indicate how various derivative estimates for solutions of nonliner second order elliptic differential equations follow directly from certain Hölder estimates by means of an interpolation technique introduced by the author in [13]. In particular, we provide alternative proofs of gradient estimates for divergence structure equations due to Ladyzhenskaya and Ural'tseva [4]; for equations in two variables due to Von Wahl [15] and for semilinear equations due to Tomi [10] and Troianiello [11].

To formulate the interpolation result, we let $\Omega$ denote a bounded domain in Euclidean $n$ space $\mathbf{R}^{n}$ and, for a vector function $w \in\left[C^{k}(\Omega)\right]^{N}, k \geqslant 0$, define the usual weighted Hölder seminorms

$$
\begin{aligned}
& |w|_{0: \Omega}=\sup _{\Omega}|w|, \\
& {[w]_{\alpha ; \Omega}^{*}=\sup _{\substack{x, y \in \Omega \\
x \neq y}} d_{x y}^{\alpha} \frac{|w(x)-w(y)|}{|x-y|^{\alpha}}, \quad 0<\alpha<1,} \\
& {[w]_{k ; \Omega}^{*}=\sup _{x \in \Omega} d_{x}^{k}\left|D^{k} w(x)\right|,} \\
& {[w]_{k, \alpha ; \Omega}^{*}=\sup _{\substack{x, y \in \Omega \\
x \neq y}} d_{x y}^{k+\alpha} \frac{\left|D^{k} w(x)-D^{k} w(y)\right|}{|x-y|^{\alpha}}, \quad 0<\alpha<1,}
\end{aligned}
$$

where $d_{x}=\operatorname{dist}(x, \partial \Omega), d_{x y}=\min \left(d_{x}, d_{y}\right)$. The following lemma now arises from the proof of the second derivative estimates in [13, Theorem 6.1].

Lemma 1. Let $w \in\left[C^{1}(\Omega)\right]^{N}$ and suppose that for any ball $B=B_{R}(y) \subset \subset \Omega$ we have

$$
[D w]_{\alpha: B}^{*} \leqslant \phi\left(M_{1} R\right)\left(1+M_{1}\right)
$$

Received by the editors October 31, 1984.

1980 Mathematics Subject Classification. Primary 35J15, 35K55; Secondary 35B45. 
where $0<\alpha<1, M_{1}=|D w|_{0 ; B}$ and $\phi$ is a nondecreasing function. Then for any $y \in \Omega, D w(y)$ can be estimated in terms of $\alpha, \phi, d_{y}$ and the modulus of continuity of $w$ on $\Omega$. In particular, for any $s>0$ and $\beta \in(0,1)$, we have the estimate

$$
[w]_{1 ; \Omega}^{*} \leqslant C\left\{s+\operatorname{diam} \Omega+s^{1-1 / \beta}\left([w]_{\beta ; \Omega}^{*}\right)^{1 / \beta}\right\}
$$

where $C$ depends on $\alpha, \beta$ and $\phi(s)$.

Proof. Since (2) is not formulated explicitly in [12], we repeat the proof details here. Suppose first that the ball $B$ is chosen so that $M_{1} R \leqslant s$, so that by (1) we have

$$
[w]_{1, \alpha ; B}^{*} \leqslant 2^{1+\alpha} \phi(s)\left(R+[w]_{1 ; \beta}^{*}\right) .
$$

Hence by the Hölder space interpolation inequality [2, Lemma 6.35], $[w]_{1 ; B}^{*}$ $\leqslant C\left(R+|w|_{0 ; B}\right)$ and, in particular, $|D w(y)| \leqslant C\left(1+R^{-1}|w|_{0 ; B}\right)$, where $C=$ $C(\alpha, \phi(s))$. By replacing $w$ by $w-w(y)$, we can assume without loss of generality that $w(y)=0$. Consequently,

$$
|w|_{0 ; B} \leqslant\left(R /\left(d_{y}-R\right)\right)^{\beta}[w]_{\beta ; \Omega}
$$

for any $\beta \in(0,1)$, and hence

$$
|D w(y)| \leqslant C\left\{1+R^{\beta-1}\left(d_{y}-R\right)^{-\beta}[w]_{\beta ; \Omega}\right\} .
$$

The proof is now completed by appropriate choice of the ball $B=B_{R}(y)$. Suppose that $[w]_{1 ; \Omega}^{*} \geqslant s$ and choose $y$ so that

$$
d_{y}|D w(y)| \geqslant[w]_{1 ; \Omega}^{*} / 2
$$

and then choose $R=s / 4|D w(y)|$. Clearly, the condition $M_{1} R \leqslant s$ is satisfied and, moreover, $d_{y} \geqslant 2 R$. Substituting for $R$ in the estimate (3) then yields (2). The first assertion is clear from inspection of the above procedure.

Let us now consider some applications.

I. Quasilinear equations. Consider second order elliptic equations of the general form

$$
Q u=a^{i j}(x, u, D u) D_{i j} u+b(x, u, D u)=0
$$

under the structure conditions: for all $x \in \Omega, M_{0}>0,|z| \leqslant M_{0}, p \in \mathbf{R}^{n}$,

$$
\begin{aligned}
& \lambda|\xi|^{2} \leqslant a^{i j}(x, z, p) \xi_{i} \xi_{j} \leqslant \Lambda|\xi|^{2} \quad \text { for all } \xi \in \mathbf{R}^{n} ; \\
& |b(x, z, p)| \leqslant \lambda\left(\mu_{0}|p|^{2}+g\right),
\end{aligned}
$$

where $\lambda, \Lambda$ and $\mu_{0} \in \mathbf{R}^{+}$and $g \in L^{n}(\Omega)$ depend on $M_{0}$. A Hölder estimate for solutions in the Sobolev space $W^{2, n}(\Omega)$ was derived in $[6,12]$, following the basic work of Krylov and Safonov [3]. This estimate may be expressed as follows:

$$
[u]_{\alpha: \Omega}^{*} \leqslant C\left(M_{0}+d\|g\|_{L^{n}(\Omega)}\right),
$$

where $M_{0}=|u|_{0: \Omega}, d=\operatorname{diam} \Omega$ and $\alpha$ and $C$ are positive constants depending only on $n, \Lambda / \lambda$ and $\mu_{0} M_{0}$. Furthermore, an estimate of the form (1) with $w=u$ and 
$\phi(t)=$ const. $(1+|t|)$ holds in each of the following cases:

(i) Equations in two variables. If $n=2$ and $g \in L^{q}(\Omega)$ for some $q>2$, then by the classical Hölder derivative estimate of Morrey (see [2, Chapter 12]),

$$
[D u]_{\alpha ; B}^{*} \leqslant K\left(1+M_{1} R\right)\left(1+M_{1}\right)
$$

where $\alpha$ depends only on $\Lambda / \lambda$ and $K$ depends on $\Lambda / \lambda, \mu_{0}, q$ and $d^{1-n / q}\|g\|_{L^{q}(\Omega)}$. Consequently, Lemma 1 provides a gradient estimate, originally due to Von Wahl [15], which we may write in the form

$$
|D u(y)| \leqslant C
$$

for any ball $B=B_{R}(y) \in \Omega$, where $C$ depends on $\Lambda / \lambda, \mu_{0} d, q, d^{1-2 / q}\|g\|_{q}$ and $\sup _{B}|u| / R$.

(ii) Equations in divergence form. Now let us assume that $Q$ has the divergence form

$$
\begin{aligned}
Q u & =\operatorname{div} A(x, u, D u)+B(x, u, D u) \\
& =A_{p_{j}}^{i} D_{i j} u+A_{z}^{i} D_{i} u+A_{x_{i}}^{i}+B
\end{aligned}
$$

and that, as well as (5) being satisfied, we have the stronger version of (6),

$$
|p|\left|A_{z}\right|,\left|A_{x}\right|,|B| \leqslant \lambda\left(\mu_{0}|p|^{2}+g\right)
$$

where $g \in L^{q}(\Omega)$ for some $q>n$. As for the two variable situation, the De Giorgi-Nash Hölder estimates for linear elliptic equations (see [2, Chapters 8, 13]) yield an estimate of the form (8) with constants $\alpha$ and $K$ depending additionally on $n$. Again we deduce immediately from Lemma 1 the gradient estimate of Ladyzhenskaya and Ural'tseva [4] for solutions $u \in W^{2,2}(\Omega)$, namely for any ball $B=B_{R}(y) \subset \Omega$,

$$
|D u(y)| \leqslant C
$$

where $C$ depends on $n, \Lambda / \lambda, \mu_{0} d, q, d^{1-n / q}\|g\|_{q}$ and $\sup _{B}|u| / R$. Other approaches to this estimate are given in $[2,5$ and 14].

(iii) Semilinear equations. For equations of the form

$$
Q u=a^{i j}(x) D_{i j} u+b(x, u, D u)=0,
$$

with principal coefficients $a^{i j} \in C^{0}(\bar{\Omega})$, satisfying (5) and (6) with $g \in L^{q}(\Omega)$, $q>n$, the estimate (8) for solutions $u \in W^{2, q}(\Omega)$ arises directly from the interior $L^{p}$ estimates and the Sobolev imbedding theorem (see [2, Theorem 7.17, 9.11]). In this case the Hölder exponent $\alpha$ is equal to $1-n / q$, if $q<\infty$, while the constant $K$ depends on $n, \Lambda / \lambda, \mu_{0}, q, d^{1-n / q}\|g\|_{q}$ and the modulus of continuity of the coefficient matrix $\left[a^{i j}\right]$. We then obtain by Lemma 1 the gradient estimate

$$
|D u(y)| \leqslant C
$$

for any ball $B=B_{R}(y) \subset \Omega$, where now $C$ depends on $n, \Lambda / \lambda, q, \mu_{0} d, d^{1-n / 4}\|g\|_{4}$, $\sup _{B}|u| / R$ and the moduli of continuity of the functions $a^{i j}$. Furthermore, by utilizing again the Hölder estimate (7), we see that (14) extends to more general equations

$$
Q u=a^{i j}(x, u) D_{i j} u+b(x, u, D u)=0
$$


with $a^{i j} \in C^{0}(\bar{\Omega} \times \mathbf{R})$. These gradient estimates correspond to those established by Tomi [10] and Troianiello [11]. In this case the application of an interpolation method was also indicated by Lieberman [8]. We also point out that, in each of the last two cases, the Hölder estimate (7) extends to $g \in L^{q}(\Omega)$ for $q>n / 2$ and, of course, by virtue of the divergence structure theory $[2,5]$, we may take $u \in L^{\infty}(\Omega) \cap$ $W^{1,2}(\Omega)$ in case (ii).

Corresponding global estimates follow by modifying the above approach at the boundary or by direct utilization of the form of the estimate (9), (12), (14), whence all we need in addition is a Lipschitz estimate at the boundary. In particular, if $\partial \Omega$ satisfies a uniform exterior sphere condition and $u=\phi$ continuously on $\partial \Omega$ for some $\phi \in C^{1,1}(\bar{\Omega})$, we infer the global estimate

$$
\sup _{\Omega}|D u| \leqslant C
$$

in each of the above cases, where $C$ depends on $n, \Lambda / \lambda, \mu_{0}, q, \Omega,\|g\|_{q}, \sup _{\Omega}|u|$ ( $\left.\leqslant M_{0}\right)$ and, in case (iii), the modulus of continuity of $\left[a^{i j}\right]$.

2. Fully nonlinear equations. We pass now to consider the estimation of second derivatives of solutions of nonlinear equations of the general form

$$
F[u]=F\left(x, u, D u, D^{2} u\right)=0
$$

under the structure conditions: for all $x \in \Omega, M_{1}>0,|z|+|p| \leqslant M_{1}, r \in \mathbf{S}^{n}$,

$$
\begin{gathered}
\lambda|\xi|^{2} \leqslant F_{r_{i j}}(x, z, p, r) \xi_{i} \xi_{j} \leqslant \Lambda|\xi|^{2} \quad \text { for all } \xi \in \mathbf{R}^{n} \\
\left|F_{x}, F_{z}(x, z, p, r)\right| \leqslant \lambda\left(\mu_{1}|r|^{2}+g_{1}\right) \\
\left|F_{p}(x, z, p, r)\right| \leqslant \lambda\left(\mu_{1}|r|+\sqrt{g_{1}}\right),
\end{gathered}
$$

where $\lambda, \Lambda, \mu_{1} \in \mathbf{R}^{+}$and $g_{1} \in L^{n}(\Omega)$ depend on $M_{1}$. Second derivative Hölder estimates may be obtained in each of the following two cases:

(i) Equations in two variables. By applying Morrey's estimate to the differentiated equation, we obtain the estimate (8) with $u$ replaced by $D u$ for $u \in C^{3}(\Omega)$ and $g_{1} \in L^{q}(\Omega)$ for some $q>2$. Consequently, Lemma 1 yields a second derivative bound for $u$ in terms of $|u|_{1, \alpha ; \Omega}$ for any $\alpha>0$. But then under the further hypothesis

$$
|F(x, z, p, 0)| \leqslant g(x), \quad g \in L^{q},
$$

for all $x \in \Omega,|z|+|p| \leqslant M_{1}$, we infer, by Morrey's estimate again,

$$
\left|D^{2} u(y)\right| \leqslant C
$$

for any ball $B=B_{R}(y) \subset \Omega$, where $C$ depends on $\Lambda / \lambda, \mu_{1}, q,\left\|g, g_{1}\right\|_{q}$, diam $\Omega$, $M_{1}=|u|_{1}$ and $\sup _{B}|D u| / R$. By means of differencing (rather than differentiation), the bound (21) extends to solutions $u \in C^{1.1}(\Omega)$.

(ii) Concave operators. In the situation considered in [13], the function $F$ was concave with respect to the $r$ variables, and its second derivatives (except for $F_{r r}$ ) 
subject to natural conditions with respect to $r$. In fact, these conditions can be combined into a single structure condition of the form

$$
\begin{aligned}
\frac{\partial^{2} F}{\partial x_{i} X_{j}} Y_{i} Y_{j} \leqslant \lambda\left\{\mu _ { 2 } \left(|r|^{3}\left|Z_{1}\right|^{2}+|r|\left(\left|Z_{2}\right|^{2}\right.\right.\right. & \left.\left.+\left|Z_{1}\right|\left|Z_{3}\right|\right)+\left|Z_{2}\right|\left|Z_{3}\right|\right) \\
& \left.+g_{2}\left(\left|Z_{1}\right|^{2}+\left|Z_{2}\right|^{2}\right)+\sqrt{g_{2}}\left|Z_{1}\right|\left|Z_{3}\right|\right\}
\end{aligned}
$$

for all $x \in \Omega,|z|+|p| \leqslant M_{1}, r \in \mathbf{S}^{n}, Y=\left(Z_{1}, Z_{2}, Z_{3}\right), Z_{1} \in \mathbf{R}^{n+1}, Z_{2} \in \mathbf{R}^{n}, Z_{3} \in$ $\mathbf{S}^{n}$, where $\lambda, \mu_{2} \in \mathbf{R}^{+}$and $g_{2} \geqslant 0 \in L^{n}(\Omega)$ depend on $M_{1}$. Following the argument of [13, Theorem (6.1)], we now conclude a Hölder bound for second derivatives of the form

$$
\left[D^{2} u\right]_{\alpha ; B}^{*} \leqslant K\left(1+M_{2} R\right)^{2}\left(1+M_{2}\right)
$$

where $M_{2}=\sup _{B}\left|D^{2} u\right|, \alpha$ depends only on $n, \Lambda / \lambda$, and $K$ depends also on $\mu_{1}, \mu_{2}$, $M_{1}=|u|_{1 ; \Omega}, q, \operatorname{diam} \Omega,\left\|g_{1}\right\|_{q ; \Omega},\left\|g_{2}\right\|_{n ; \Omega}$ for $q>n$. Hence, Lemma 1 yields an interior bound for $D^{2} u$ in terms of $[D u]_{\beta ; \Omega \Omega}^{*}$ for $\beta>0$. The latter quantity can be a priori bounded [13, Theorem 5.1] if we restrict (19) slightly, by requiring also

$$
\left|F_{z}\right|,\left|F_{x}\right| \leqslant \lambda\left(\varepsilon|r|^{2}+\tilde{g}\right)
$$

where $\tilde{g} \in L^{n}(\Omega)$ and $\varepsilon$ is a positive number depending only on $n, \Lambda / \lambda, M_{1}$. Consequently, under the structural hypotheses (18), (19), (22), (24), we obtain for solutions $u \in C^{2}(\Omega)$, an interior estimate

$$
\left|D^{2} u(y)\right| \leqslant C
$$

for any ball $B=B_{R}(y) \subset \Omega$, where $C$ depends on $\Lambda / \lambda, n, \mu_{1}, \mu_{2}$, diam $\Omega, q,\left\|g_{1}\right\|_{q}$, $\left\|\tilde{g}, g_{2}\right\|_{n}, M_{1}\left(=|u|_{1}\right)$ and $\sup _{B}|D u| / R$. The bound (25) also extends to solutions $u \in C^{1,1}(\Omega)$.

We point out that if (20) holds for bounded $g$, then the full gradient $D u$ may be replaced by $D^{\prime} u=\left(D_{1} u, \ldots, D_{n-1} u\right)$ in the quantity $\sup _{B}|D u| / R$ in the estimates (21), (25). As a result, these estimates extend to global second derivative estimates if, for example, $\partial \Omega \in C^{3}$ and $u / \partial \Omega \in C^{3}$ [13, Theorem 7.3]. Conditions ensuring interior and global gradient bounds, as well as other approaches to (25), are treated in the papers $[1,9,13]$.

Finally, let us remark that the interpolation lemma can be readily extended to cover parabolic problems, with the above estimates all having parabolic analogues. Also, it may be used to deduce global derivative bounds under appropriate oblique derivative boundary conditions (as considered, for example, in $[5,7,9]$ ) in each of the above situations.

\section{REFERENCES}

1. L. Caffarelli, J. J. Kohn, L. Nirenberg and J. Spruck, The Dirichlet problem for nonlinear second order elliptic equations. II. Complex Monge-Ampère and uniformly elliptic equations, Comm. Pure Appl. Math. (to appear).

2. D. Gilbarg and N. S. Trudinger, Elliptic partial differential equations of second order (2nd ed.), Springer-Verlag, Berlin, Heidelberg, New York, 1983. 
3. N. V. Krylov and M. V. Safonov, Certain properties of solutions of parabolic equations with measurable coefficients, Izv. Akad. Nauk SSSR 40 (1980), 161-175. English transl., Math. USSR-Izv. $16(1981), 151-164$

4. O. A. Ladyzkenskaya and N. N. Ural'tseva, On the smoothness of quasilinear equations in several variables and of variational problems, Comm. Pure Appl. Math. 14 (1961), 481-495.

5. __ Linear and quasilinear elliptic equations, Academic Press, New York, 1968.

6. __ Hölder estimates for solutions of second order quasilinear elliptic equations in general form, Zap. Nauchn. Sem. Leningrad. Otdel. Mat. Inst. Steklov (LOMI) 96 (1980), 161-168. English transl., J. Soviet Math. 21 (1983), 762-768.

7. G. M. Lieberman, The conormal derivative problem for elliptic equations of variational type, J. Differential Equations 49 (1983), 218-257.

8. __ Gradient estimates for semilinear elliptic equations, Proc. Royal Soc. of Edinburgh (to appear)

9. G. M. Lieberman and N. S. Trudinger, Nonlinear oblique boundary value problems for nonlinear elliptic equations, Austral. Nat. Univ. Centre for Math. Anal. Report R24, 1984.

10. F. Tomi, Über semilineare elliptische Differentialgleichungen zweiter Ordnung, Math. Z. 111 (1969), 350-366.

11. G. M. Troianiello, Maximal and minimal subsolutions to a class of elliptic quasilinear problems, Proc. Amer. Math. Soc. 91 (1984), 95-102.

12. N. S. Trudinger, Local estimates for subsolutions and supersolutions of general second order elliptic quasilinear equations, Invent. Math. 61 (1980), 67-79.

13. __ Fully nonlinear, uniformly elliptic equations under natural structure conditions, Trans. Amer. Math. Soc. 278 (1983), 751-769.

14. On the estimation of derivatives of solutions of second order elliptic equations, in preparation.

15. W. Von Wahl, Über quasilineare elliptische Differentialgleichungen in der Ebene, Manuscripta Math. 8 (1973), 59-67.

Centre for Mathematical Analysis, Australian National University, G. P. O. Box 4, Canberra A. C. T. 2600, Australia 